\title{
Effects of the antihypertensive drug benidipine on osteoblast function in vitro
}

\author{
BAIXIANG WANG ${ }^{1}$, MING BI $^{2 *}$, ZHEN ZHU ${ }^{3}$, LEI WU ${ }^{1}$ and JINGYUN WANG ${ }^{1 *}$ \\ Departments of ${ }^{1}$ Prosthodontics and ${ }^{2}$ Comprehensive Treatment, School of Stomatology, Jilin University, \\ Changchun, Jilin 130021; ${ }^{3}$ Department of Prosthodontics, Zhenjiang Stomatological Hospital, \\ Zhenjiang, Jiangsu 212002, P.R. China
}

Received September 4, 2013; Accepted December 27, 2013

DOI: $10.3892 /$ etm.2014.1475

\begin{abstract}
The dihydropyridine-type calcium channel blocker, benidipine (BD) has been widely used in hypertension therapy. Previous studies have demonstrated that BD has a positive effect on bone metabolism. Inspired by this promoting phenomenon, the present study investigated the effects of BD on osteoblasts in vitro. Experiments were designed and performed, including an MTT assay, reverse transcription-polymerase chain reaction, western blot analysis, alkaline phosphatase activity measurements and alizarin red $\mathrm{S}$ staining. The results demonstrated that $\mathrm{BD}$ promoted osteoblast proliferation and osteogenic differentiation at concentrations from $1 \times 10^{-6}$ to $1 \times 10^{-9} \mathrm{M}$ by upregulating Runx2, BMP2 and OCN gene expression levels. Overall, BD at appropriate concentrations has been demonstrated to have positive effects on osteoblast function in addition to its conventional clinical usage.
\end{abstract}

\section{Introduction}

As one of the most common types of chronic disorder in aged people, osteoporosis has a multifactorial etiology and has been characterized by progressive bone substance loss, microarchitecture impairments and an increased risk of fractures $(1,2)$. In addition to the systemic symptoms, patients with osteoporosis also suffer from the dental diseases periodontitis and dentition defect, which usually cause bone mass insufficiency $(3,4)$. Due to impaired alveolar bone structure and metabolic disturbances, it is accordingly difficult to treat these patients. A great deal of effort has been made to alleviate this situation, yet few treatment technologies are applied widely. In current clinical practice, most dentists prefer to select drugs as facilitators (5).

Correspondence to: Professor Jingyun Wang, Department of Prosthodontics, School of Stomatology, Jilin University, 1500 Qinghua Road, Changchun, Jilin 130021, P.R. China

E-mail: jlccjingyun@gmail.com

*Contributed equally

Key words: benidipine, calcium channel blockers, osteoporosis, hypertension
Osteoblasts usually originate from mesenchymal stem cells and are of importance during the bone formation process (6). Osteoblasts often behave abnormally in bone metabolism disorders. Therefore, numerous drugs for osteoporosis treatment are targeted at regulating osteoblast function.

Osteoblasts express several types of calcium channels. Of these channels, the L-type voltage-sensitive channel is the one most clearly involved in functional osteoblast regulation. A previous study has demonstrated that calcium channels are associated with proliferation, apoptosis and differentiation in osteoblasts (7). As a large number of patients who suffer from bone metabolism disorders also require hypertension treatment, the identification an appropriate antihypertensive drug that is able to also treat bone disorders would be of great significance. If a drug stimulates osteoblast function while performing an antihypertensive effect, it is likely to present a great benefit for elderly patients and doctors.

Benidipine (BD) is a dihydropyridine-type calcium channel blocker and has been widely used for hypertension therapy. It blocks the L-type and T-type calcium channels in different types of cells, including osteoblasts (8). Due to the dual effects of BD on hypertension and calcium channels, it is hypothesized to be a suitable candidate for the treatment of patients with osteoporosis and hypertension. Therefore, the aim of the present study was to evaluate the effect of BD at different concentrations on osteoblasts in vitro.

\section{Materials and methods}

Medicine preparation. A solution of BD (Kyowa Hakko Kirin Co., Ltd., Tokyo, Japan) was prepared by dissolving solid BD in dimethylsulfoxide (DMSO) solvent. The stock solution was stored at $-20^{\circ} \mathrm{C}$.

Cell culture. MC3T3-E1 cells (American Type Culture Collection,Manassas, VA, USA) were cultured in $\alpha$-MEM containing $100 \mathrm{U} / \mathrm{ml}$ penicillin, $100 \mathrm{U} / \mathrm{ml}$ streptomycin and $10 \%$ fetal bovine serum (FBS) in a humidified incubator at $37^{\circ} \mathrm{C}$ and $5 \% \mathrm{CO}_{2}$. The cells were subcultured every three days in the presence of $0.25 \%$ trypsin.

MTT assay. MC3T3-E1 cells were seeded in 96-well plates $(5,000$ cells/well) and incubated overnight. BD solu- 
Table I. Primer sequences used for RT-PCR.

\begin{tabular}{lll}
\hline Gene & \multicolumn{1}{c}{ Forward (5'-3') } & \multicolumn{1}{c}{ Reverse (5'-3') } \\
\hline Runx2 & TTCTCCAACCCACGAATGCAC & CAGGTACGTGTGGTAGTGAGT \\
BMP2 & TGGCCCATTTAGAGGAGAACC & AGGCATGATAGCCCGGAGG \\
OCN & GAACAGACTCCGGCGCTA & AGGGAGGATCAAGTCCCG \\
GAPDH & GACTTCAACAGCAACTCCCAC & TCCACCACCCTGT TGCTGTA
\end{tabular}

RT-PCR, reverse transcription-polymerase chain reaction.

tion at different concentrations (final concentrations of $1 \times 10^{-4}-1 \times 10^{-10} \mathrm{M}$ ) was then added. Cells without BD treatment were used as a negative control and wells without cells were set as blanks. One-, two-, and three-day further incubations were performed, and then $20 \mu \mathrm{l}$ MTT $(5.0 \mathrm{mg} / \mathrm{ml})$ was added and the cells were incubated for another $4 \mathrm{~h}$ at $37^{\circ} \mathrm{C}$. Subsequently, the supernatant was removed, DMSO was added and the optical density (OD) at $570 \mathrm{~nm}$ was measured on a microplate spectrophotometer (Model 680 Microplate Reader; Bio-Rad, Hercules, CA, USA). The proliferation rate of the cells was calculated according to the following formula: $\left(\mathrm{OD}_{\text {sample }}-\mathrm{OD}_{\mathrm{b}}\right.$ lank $) /\left(\mathrm{OD}_{\text {control }}-\mathrm{OD}_{\text {blank }}\right)$.

Assay for alkaline phosphatase (ALP) activity. MC3T3-E1 cells were seeded in 24 -well plates $\left(2 \times 10^{4}\right.$ cells/well $)$ containing $\alpha$-MEM medium and 10\% FBS. After $24 \mathrm{~h}$, the culture medium was changed to $\alpha$-MEM, $10 \%$ FBS and osteogenic induction supplement containing $10 \mathrm{mmol} / \mathrm{l}$ disodium $\beta$-glycerophosphate and $0.15 \mathrm{mmol} / \mathrm{l}$ ascorbic acid (Sigma, St. Louis, MO, USA). A series of dilutions of BD (final concentrations, $1 \times 10^{-6}-1 \times 10^{-9} \mathrm{M}$ ) were added to the culture medium in the 24-well plates for 3, 5, 7, 10 and 14 days. MC3T3-E1 cells treated with only osteogenic induction supplement were used as the control group. Following incubation, the MC3T3-E1 cells were washed twice with ice-cold PBS and lysed by two cycles of freezing and thawing. Aliquots of the supernatants were subjected to ALP activity and protein content measurement using an ALP activity kit and a bicinchonininc acid (BCA) protein assay kit (Nanjing Jiancheng Bioengineering Institute, Nanjing, China). All the results were normalized by protein content.

Assay for mineralized matrix formation. Cells were seeded in 24-well plates $\left(2 \times 10^{4}\right.$ cells/well $)$ and cultured overnight at $37^{\circ} \mathrm{C}$ in a $5 \% \mathrm{CO}_{2}$ humidified incubator. The medium was then changed to medium containing osteogenic induction supplement and BD $\left(1 \times 10^{-6}-1 \times 10^{-9} \mathrm{M}\right)$ for 21 days. The formation of mineralized matrix nodules was determined by alizarin red $\mathrm{S}$ (ARS) staining. Briefly, the cells were fixed in $95 \%$ ethanol for $30 \mathrm{~min}$ at room temperature. The fixed cells were washed with PBS and stained with $1 \%$ ARS (pH 4.2) for 30 min at room temperature. Quantitative analysis was performed by elution with $10 \%(\mathrm{w} / \mathrm{v})$ cetylpyridium chloride for $10 \mathrm{~min}$ at room temperature, and the OD was measured at $570 \mathrm{~nm}$.

$R N A$ isolation and semiquantitative reverse transcription-polymerase chain reaction (RT-PCR). Total RNA was extracted following three-day incubation using TRIzol reagent (Invitrogen Life Technologies, Carlsbad, CA, USA). Complementary DNA (cDNA) was produced using a transcriptase PCR kit (ReverTra Dash; Toyobo Biochemicals, Osaka, Japan). Aliquots of total cDNA were amplified, using PCR equipment (PC701 thermal cycler; Astec, Fukuoka, Japan). The amplification reaction products were resolved on $1.5 \%$ agarose/TAE gels by electrophoresis at $100 \mathrm{mV}$, and were visualized by ethidium-bromide staining. The primers used are presented in Table I.

Western blot analysis. MC3T3-E1 cells were washed with cold PBS and lysed in cold Tris- $\mathrm{HCl}(50 \mathrm{mM}, \mathrm{pH} 7.4), 10 \mathrm{mM}$ EDTA, 4.3 $\mathrm{M}$ urea and $1 \%$ Triton X-100. Proteins were subjected to SDS-PAGE using $10 \%$ separation gel and transferred to a nitrocellulose membrane. The membrane was blocked for $2 \mathrm{~h}$ at room temperature with $5 \%$ bovine serum albumin in TBST solution (10 mM Tris- $\mathrm{HCl}, \mathrm{pH} 8.0 ; 150 \mathrm{mM} \mathrm{NaCl} ; 0.05 \%$ Tween-20). Subsequently, the blots were incubated with the corresponding primary antibodies (rabbit anti-Runx2, rabbit anti-BMP2 and rabbit anti-OCN) (Biosynthesis Biotechnology Co.,Ltd., Beijing, China) in the TBST solution overnight at $4^{\circ} \mathrm{C}$, followed by $2 \mathrm{~h}$ incubation with secondary goat anti-rabbit IgG antibodies (Santa Cruz Biotechnology Inc., Santa Cruz, CA, USA) conjugated with horseradish peroxidase, and visualized with an enhanced luminol-based chemiluminescent (ECL) kit (Thermo Fisher Scientific Inc., Waltham, MA, USA). The OD of the bands was quantified using LAS-1000 luminescent image analyzer software (Fujifilm, Berlin, Germany).

Statistical analysis. One-way analysis of variance and Tukey's multiple comparison tests were performed to detect any significant effects that occurred as a result of the experimental variables. All results are expressed as the mean \pm standard deviation. $\mathrm{P}<0.05$ was considered to indicate a statistically significant difference.

\section{Results}

Effect of BD on the proliferation of MC3T3-E1 cells. As demonstrated in Fig. 1B, the effect of BD on the proliferation of MC3T3-E1 cells was time-dependent and the proliferation rate decreased with increasing $\mathrm{BD}$ concentrations. Following one-, two- and three-day treatment, BD promoted proliferation at concentrations of $1 \times 10^{-6}-1 \times 10^{-9} \mathrm{M}$. The higher concentrations of $\mathrm{BD}$ inhibited cell proliferation whereas no significant difference from the control was observed when the lower concentrations of BD were applied. 
A

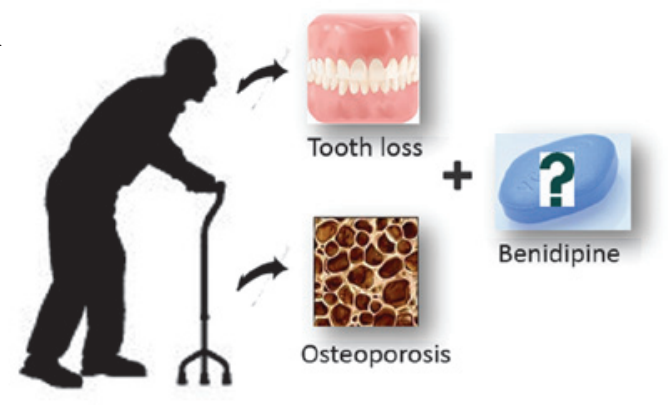

C

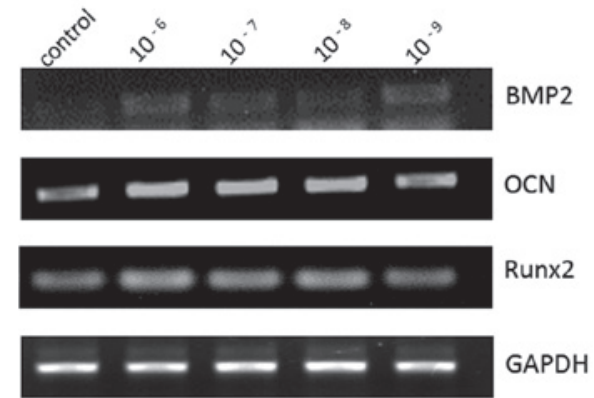

B
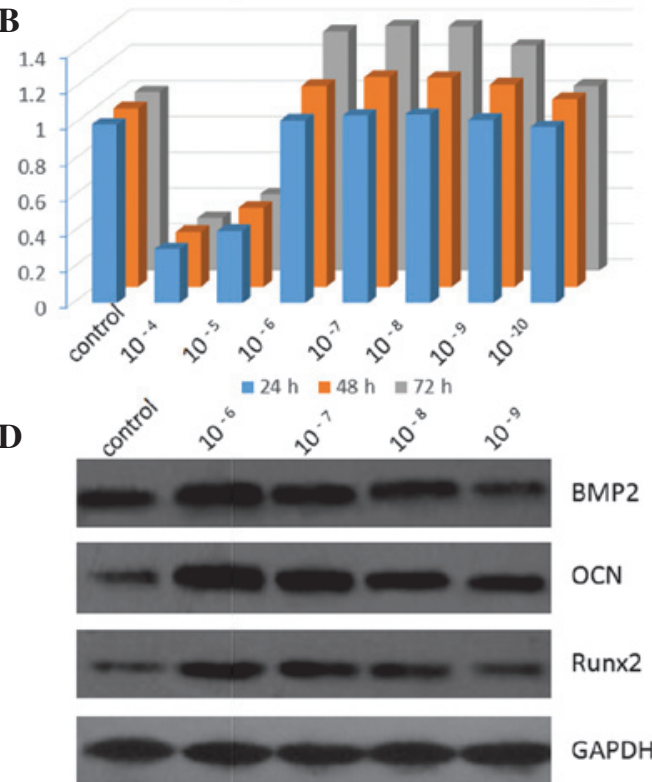

Figure 1. (A) Schematic diagram demonstrating that patients with osteoporosis suffer from systemic and dental symptoms. (B) The osteoblast proliferation rate is increased in the presence of $\mathrm{BD}$ at certain concentrations over a three day period. Inhibition of proliferation was observed when higher concentrations of $\mathrm{BD}$ were applied to the cells. Data are presented as the mean \pm SD. (C) Gene and (D) protein expression levels of BMP2, OCN and Runx2 were upregulated when MC3T3-E1 cells were treated with BD for three days. BD, Benidipine.

A

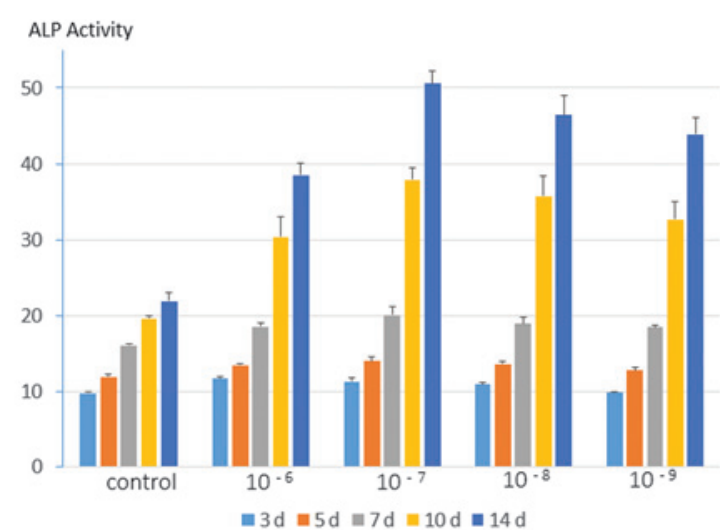

B

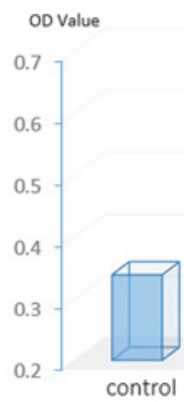

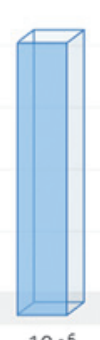

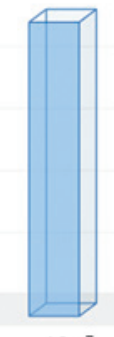

$10^{\cdot 7}$

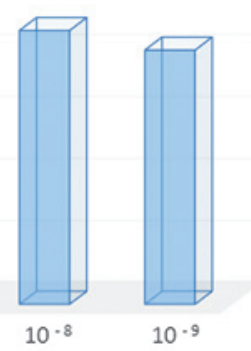

$10 \cdot 9$

C
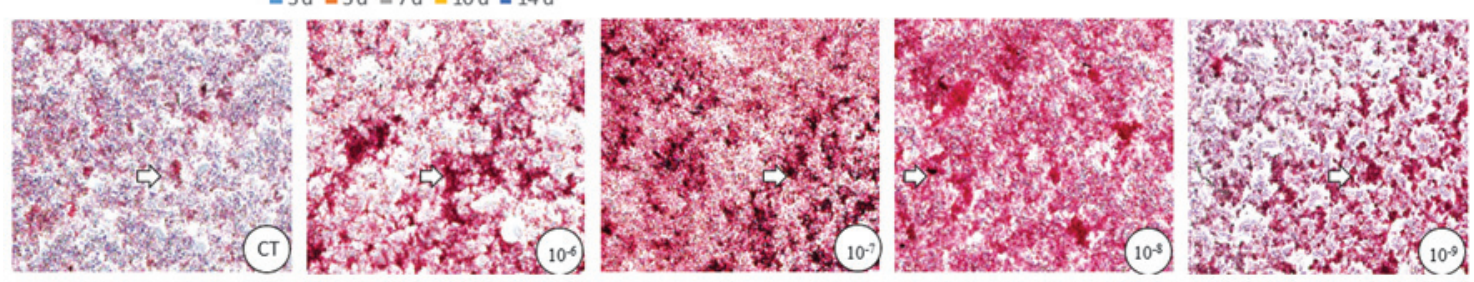

Figure 2. (A) ALP activity was enhanced significantly in a time-dependent manner when osteoblasts were treated with BD. (B) Mineralization nodules were dissolved by cetylpyridium chloride and the OD value was measured at $570 \mathrm{~nm}$. The results demonstrated prominent enhancement of mineralization when $\mathrm{BD}$ was applied. (C) ARS staining was conducted following 21-day osteogenic induction. The white arrows indicate the mineralization nodules. Data are presented as the mean $\pm \mathrm{SD}$. ALP, alkaline phosphatase; OD, optical density; CT, control; BD, Benidipine; ARS, Alazarin red S.

Effect of BD on gene and protein expression. Following treatment with different concentrations of $\mathrm{BD}$ for three days, BMP2, OCN and Runx2 mRNA levels were markedly upregulated compared with those in the control group in a concentration-dependent manner (Fig. 1C). As demonstrated in Fig. 1D, BMP2, OCN and Runx2 protein levels were enhanced following BD treatment; the most prominent enhancements were observed in the groups of cells treated with $1 \times 10^{-6}$ and $1 \times 10^{-7} \mathrm{M}$ BD.
Effect of BD on the differentiation of MC3T3-E1 cells. As presented in Fig. 2A, all four groups treated with BD exhibited elevated levels of ALP activity compared with those in the control group. The highest level was observed in the cells treated with $1 \times 10^{-7} \mathrm{M}$ BD. From day 3 to day 14, the level of ALP activity in each of the groups of cells increased in a time-dependent manner.

BD also promoted the formation of mineralized matrix nodules in the MC3T3-E1 cells. The results of the quantita- 
tive analysis of the ARS staining were in accordance with the morphological observations. $\mathrm{BD}$ at $1 \times 10^{-7} \mathrm{M}$ also resulted in the clearest promotive effect in the cells (Fig. 2B and C).

\section{Discussion}

Cell proliferation is a key attribute of the bone repair process. In the present study, it was demonstrated that the effects of $\mathrm{BD}$ on the proliferation of MC3T3-E1 cells varied according to the concentration of $\mathrm{BD}$. A low concentration of $\mathrm{BD}$ had no effect on cell proliferation. At a BD concentration of $1 \times 10^{-6} \mathrm{M}$, cell proliferation was promoted and this effect was observed in the cells treated with concentrations down to $1 \times 10^{-9} \mathrm{M} \mathrm{BD}$, whereas inhibition was observed when cells were treated with the higher concentrations. Thus, the concentrations $1 \times 10^{-6}-1 \times 10^{-9} \mathrm{M}$ BD were selected for the remaining experiments. The result is inconsistent with a previous study (9) and this may be attributable to the different techniques applied. The inhibitory effect is likely to be the result of cytotoxicity.

A large number of proteins that have been associated with bone cells are specifically required for osteoblast differentiation, such as Runx2, BMP2 and OCN (10). Runx2 is a master regulator of osteogenic gene expression and osteoblast differentiation. It has been reported that Runx 2 knockout mice exhibit no bone tissues or osteoblasts, indicating that osteoblast differentiation is completely blocked in the absence of Runx2 (11,12). In addition to being required for osteoblast differentiation, Runx 2 is necessary for the proper function of mature osteoblasts, including the synthesis of bone matrix (13).

$\mathrm{OCN}$ is the most specific gene for osteoblast differentiation and mineralization. OCN is expressed during the postproliferative period, reaches its maximum expression during mineralization and accumulates in the mineralized bone (14). BMP2 is a member of the transforming growth factor- $\beta$ superfamily and has a key regulatory role as a cell-cell signaling molecule during bone formation and repair. BMP2, which is a potent osteogenic protein required for osteoblast differentiation and bone formation, induces low levels of expression of osteoblast marker genes such as OCN and ALP in calvarial cells from $\mathrm{Cbfa1}^{-/-}$animals (12). Furthermore, it has been shown that mice lacking BMP2 in the limb mesenchyme exhibit a clear defect in bone mineral density shortly after birth, indicating that BMP2 has a unique role in bone formation (15).

ALP, a cell membrane-associated enzyme, appears early during osteoblast differentiation and is the most widely recognized marker of osteoblastic differentiation (16). ALP activity correlates with matrix formation in osteoblasts prior to the initiation of mineralization. In the present study, BD enhanced ALP activity at five time points in a time-dependent manner, but no significant concentration-dependent manner was observed. While the appearance of ALP activity is an early marker of differentiation, mineralized nodule formation is considered as a late marker for maturation $(17,18)$. Consistent with the ALP activity result, an increased mineralization level was observed in the BD-treated cells. This was further confirmed by the quantitative analysis.

In addition to the conventional antihypertensive function of $\mathrm{BD}$, several studies have suggested that BD increases the ALP activity of osteoblastic cells and also stimulates mineral matrix deposition $(19,20)$. In addition, BD has been shown to decrease receptor activator of nuclear factor $\mathrm{\kappa B}$ ligand expression in human osteoblasts, indicating the suppression of osteoclast differentiation (21). The systematic experiments conducted in the present study also demonstrated that BD promoted the proliferation, osteogenic differentiation and mineralization of MC3T3-E1 cells at the cellular and molecular levels, when applied at concentrations of $1 \times 10^{-6}-1 \times 10^{-9} \mathrm{M}$. These findings indicate that BD may be a novel candidate for the combined treatment of osteoporosis and hypertension. BD promoted osteogenesis most markedly at concentrations of $1 \times 10^{-7}$ and $1 \times 10^{-8} \mathrm{M}$, which is in accordance with the serum drug levels for antihypertensive therapy (22).

The results of the present study demonstrated that $\mathrm{BD}$ promotes cell proliferation and osteogenic differentiation at concentrations from $1 \times 10^{-6}$ to $1 \times 10^{-9} \mathrm{M}$ by upregulating Runx2, BMP2 and OCN gene expression levels. Therefore, it was concluded that $\mathrm{BD}$ at the appropriate concentrations may have a positive effect on osteoblast function in addition to its conventional usage, and may be a suitable candidate for the treatment of patients with osteoporosis and hypertension.

\section{References}

1. Warriner AH and Saag KG: Osteoporosis diagnosis and medical treatment. Orthop Clin North Am 44: 125-135, 2013.

2. Mosekilde L, Vestergaard P and Rejnmark L: The pathogenesis, treatment and prevention of osteoporosis in men. Drugs 73: 15-29, 2013.

3. Bertulucci Lde A, Pereira FM, de Oliveira AE, Brito LM and Lopes FF: Periodontal disease in women in post-menopause and its relationship with osteoporosis. Rev Bras Ginecol Obstet 34: 563-567, 2012 (In Portuguese).

4. Alania KN, Iverieli MB, Abashidze NO, Gogishvili KhB and Chigladze TT: Oral cavity features in patients suffering from osteogenesis imperfecta. Georgian Med News 193: 34-41, 2011 (In Russian).

5. Lippuner K: The future of osteoporosis treatment - a research update. Swiss Med Wkly 142: w13624, 2012.

6. Teti A: Bone development: overview of bone cells and signaling. Curr Osteoporos Rep 9: 264-273, 2011.

7. Blair HC, Schlesinger PH, Huang CL and Zaidi M: Calcium signalling and calcium transport in bone disease. Subcell Biochem 45: 539-562, 2007.

8. Yao K, Nagashima K and Miki H: Pharmacological, pharmacokinetic, and clinical properties of benidipine hydrochloride, a novel, long-acting calcium channel blocker. J Pharmacol Sci 100: 243-261, 2006.

9. Kosaka N and Uchii M: Effect of benidipine hydrochloride, a dihydropyridine-type calcium antagonist, on the function of mouse osteoblastic cells. Calcif Tissue Int 62: 554-556, 1998.

10. Ducy P, Zhang R, Geoffroy V, Ridall AL and Karsenty G: Osf2/Cbfa1: a transcriptional activator of osteoblast differentiation. Cell 89: 747-754, 1997.

11. Nakashima K, Zhou X, Kunkel G, et al: The novel zinc finger-containing transcription factor osterix is required for osteoblast differentiation and bone formation. Cell 108: 17-29, 2002.

12. Komori T, Yagi H, Nomura S, et al: Targeted disruption of Cbfa1 results in a complete lack of bone formation owing to maturational arrest of osteoblasts. Cell 89: 755-764, 1997.

13. Ducy P, Starbuck M, Priemel M, et al: A Cbfa1-dependent genetic pathway controls bone formation beyond embryonic development. Genes Dev 13: 1025-1036, 1999.

14. Neve A, Corrado A and Cantatore FP: Osteocalcin: skeletal and extra-skeletal effects. J Cell Physiol 228: 1149-1153, 2013.

15. Tsuji K, Bandyopadhyay A, Harfe BD, et al: BMP2 activity, although dispensable for bone formation, is required for the initiation of fracture healing. Nat Genet 38: 1424-1429, 2006.

16. Beertsen W and Van den Bos T: Alkaline phosphatase induces the deposition of calcified layers in relation to dentin: an in vitro study to mimic the formation of afibrillar acellular cementum. J Dent Res 70: 176-181, 1991 
17. Liu D, Zhang J, Wang G, Liu X, Wang S and Yang M: The dual-effects of $\mathrm{LaCl}_{3}$ on the proliferation, osteogenic differentiation, and mineralization of MC3T3-E1 cells. Biol Trace Elem Res 150: 433-440, 2012.

18. Liu D, Zhang J, Li Y, Wang S and Yang M: The effects of Ce on the proliferation, osteogenic differentiation and mineralization function of MC3T3-E1 cells in vitro. Biol Trace Elem Res 149: 291-297, 2012

19. Nishiya Y, Kosaka N, Uchii M and Sugimoto S: A potent 1,4-dihydropyridine L-type calcium channel blocker, benidipine, promotes osteoblast differentiation. Calcif Tissue Int 70: 30-39, 2002.

20. Nishiya $Y$ and Sugimoto S: Effects of various antihypertensive drugs on the function of osteoblast. Biol Pharm Bull 24: 628-633, 2001.
21. Shimizu H, Nakagami H, Yasumasa N, Mariana OK, Kyutoku M, Koriyama H, Nakagami F, Shimamura M, Rakugi $H$ and Morishita R: Links between hypertension and osteoporosis: benidipine ameliorates osteoporosis in ovariectomized hypertensive rats through promotion of osteoblast proliferation and inhibition of osteoclast differentiation. Curr Cardiovasc Risk Rep 6: 274-280, 2012.

22. Yun HY, Yun MH, Kang W and Kwon KI: Pharmacokinetics and pharmacodynamics of benidipine using a slow receptor-binding model. J Clin Pharm Ther 30: 541-547, 2005. 